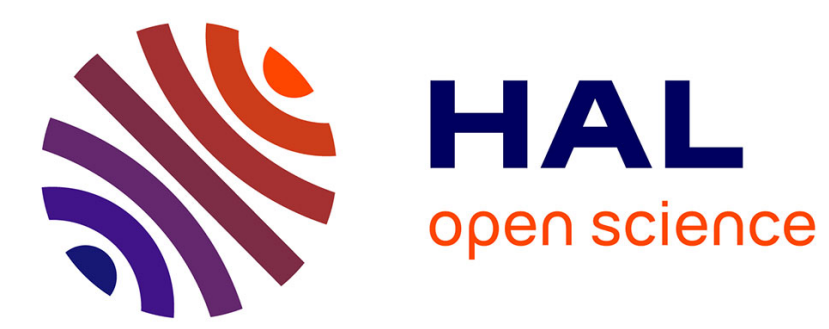

\title{
Canonical instabilities of autonomous vehicle systems
}

Rodrick Wallace

\section{To cite this version:}

Rodrick Wallace. Canonical instabilities of autonomous vehicle systems. 2016. hal-01275653

\section{HAL Id: hal-01275653 \\ https://hal.science/hal-01275653}

Preprint submitted on 17 Feb 2016

HAL is a multi-disciplinary open access archive for the deposit and dissemination of scientific research documents, whether they are published or not. The documents may come from teaching and research institutions in France or abroad, or from public or private research centers.
L'archive ouverte pluridisciplinaire HAL, est destinée au dépôt et à la diffusion de documents scientifiques de niveau recherche, publiés ou non, émanant des établissements d'enseignement et de recherche français ou étrangers, des laboratoires publics ou privés.

\section{다)(1) $(5$}

Distributed under a Creative Commons Attribution - NonCommerciall 4.0 International 


\title{
Canonical instabilities of autonomous vehicle systems
}

\author{
Rodrick Wallace \\ Division of Epidemiology \\ The New York State Psychiatric Institute*
}

February 3, 2016

\begin{abstract}
Formal argument suggests that command, communication and control systems can remain stable in the sense of the Data Rate Theorem that mandates the minimum rate of control information required to stabilize inherently unstable 'plants', but may nonetheless, under fog-of-war demands, collapse into dysfunctional modes at variance with their fundamental mission. We apply the theory to autonomous ground vehicles under intelligent traffic control in which swarms of interacting, self-driving devices are inherently unstable as a consequence of the basic irregularity of the road network. It appears that such 'V2V/V2I' systems will experience large-scale failures analogous to the vast propagating fronts of power network blackouts, and possibly less benign, but more subtle patterns of 'psychopathology' at various scales.
\end{abstract}

Key Words: control theory; driving experience; groupoid; information theory; turbulence; vehicle density

...[T]he psychological actions of drivers make traffic different from any other flow (Orosz et al. 2010).

...[A]ll models are wrong, but some are useful (Box and Draper 1987)

\section{Introduction}

We use a variety of mathematical models to explore the dynamics of rapidacting, inherently unstable command, communication and control systems $\left(C^{3}\right)$ that are cognitive in the sense that they must, in an appropriate 'real time', evaluate a large number possible actions and choose a small subset for implementation. Such choice decreases uncertainty, in a precise formal manner, and

*Wallace@nyspi.columbia.edu,rodrick.wallace@gmail.com 
reduction in uncertainty implies the existence of an information source. See Wallace $(2012,2015 \mathrm{a})$ for more details. We first examine dynamics related to V2V/V2I automated ground vehicle systems from the perspective of the Data Rate Theorem (e.g., Nair et al. 2007), extend the argument to more general phase transition analogs for $C^{3}$ structures, study instability as onset of 'turbulent' modes, and then use an information bottleneck method to derive similar results, developing statistical tools useful at different scales and levels of organization. V2V/V2I atuomated ground vehicle systems operate along geodesics in a densely convoluted 'map quotient space' - more fully described later - that is in contrast to the problem of air traffic control, where locally stable vehicle paths are seen as thick braid geodesics in a simpler Euclidean quotient space (Hu et al. 2001). Such geodesics are generalizations of the streamline characteristics of hydrodynamic flow (e.g., Landau and Lifshitz 1987).

\section{Data Rate Theorem}

Unlike aircraft, which can be constructed to be inherently stable in linear flight by placing the aerodynamic center of pressure sufficiently behind the mechanical center of gravity, the complex nature of road geometry and density of dense vehicular traffic ensures that V2V/V2I systems will be inherently unstable, requiring constant input of control information to prevent, at the least, traffic jams and tie-ups. For a linear 'plant' described by an n-dimensional state vector $x_{t}$ at time $t$, the dynamics are given by the vector equation

$$
x_{t+1}=A x_{t}+\text { Stochastic and Control terms }
$$

where $A$ is an appropriate $n \times n$ system matrix.

Let $\mathcal{H}$ be the control information rate needed to stabilize a rapidly-responding but inherently unstable $C^{3}$ system. By the Data Rate Theorem (DRT), $\mathcal{H}$ must be greater than the rate at which 'topological information' is generated. For the linear system of Eq.(1), that rate is

$$
\alpha \equiv \log \left[\left|\operatorname{det}\left(A^{u}\right)\right|\right]
$$

where det is the determinant and $A^{u}$ is the decoupled component of the matrix $A$ having eigenvalues $\geq 1$ (Nair et al. 2007).

For a fixed road network, it is evident that the central topological variate is the vehicle density that defines system dynamics under the 'fundamental diagram' relating vehicular flow per unit time to vehicle density per unit length. See figure (1), showing, for a Rome street, the number of vehicles/hour as a function of vehicles/mile. Behavior shifts from regular to 'uncontrolled' at about $40 \mathrm{v} / \mathrm{mi}$.

Under such circumstances, vehicle density $\rho$ is the only parameter defining the rate of topological information generation, and we can write the stability relation of the DRT as

$$
\mathcal{H}>\alpha=f(\rho) \alpha_{0}
$$




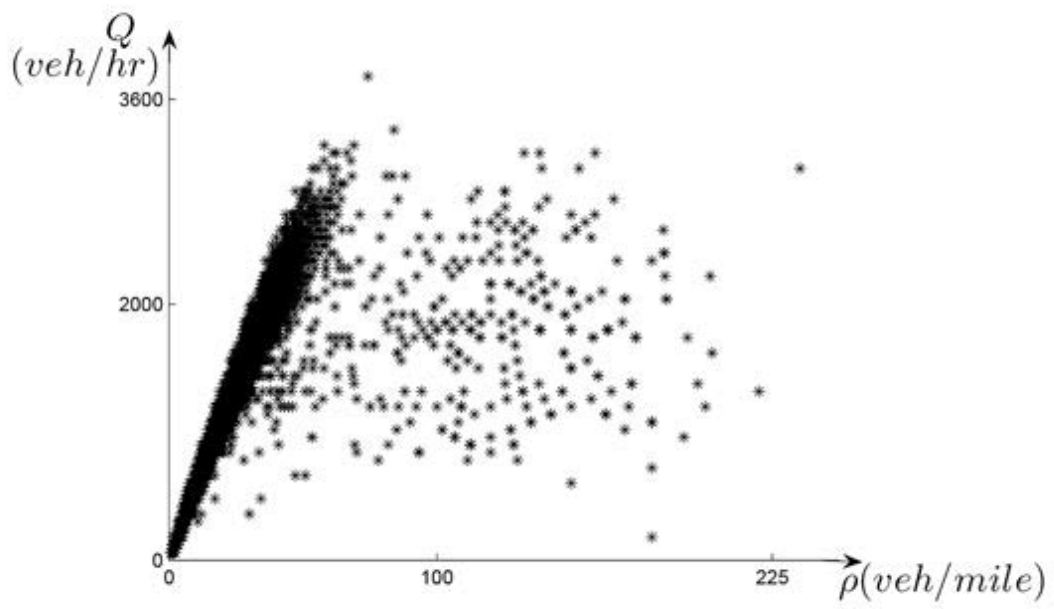

Figure 1: Vehicles per hour as a function of vehicle density per mile for a street in Rome (Blandin et al. 2011). Both streamline geodesic flow and the phase transition to 'crystallized' turbulent flow at critical traffic density are evident. Some of the states may be 'supercooled', i.e., delayed 'crystallization' in spite of high traffic density. 'Fine structure' can be expected within both geodesic and turbulent modes. 
where $\alpha_{0}$ is a road network constant and $f(\rho)$ is a positive, monotonically increasing function. The Mathematical Appendix uses a classic Black-Scholes argument to approximate the 'cost' of $\mathcal{H}$ as a function of the 'investment' $\rho$ as a linear function. Similarly, we can, again in first order, approximate $f(\rho)$ as a linear function, giving the limit condition for stability as

$$
\frac{\kappa_{1} \rho+\kappa_{2}}{\kappa_{2} \rho+\kappa_{4}}>\alpha_{0}
$$

For $\rho=0$ the stability condition is $\kappa_{2} / \kappa_{4}>\alpha_{0}$. At large $\rho$ this becomes $\kappa_{1} / \kappa_{2}>\alpha_{0}$. If $\kappa_{2} / \kappa_{4} \gg \kappa_{1} / \kappa_{2}$, the stability condition may be violated at high traffic density, and instability becomes manifest, as at higher densities in figure (1).

A more complex model, based explicitly on the cognitive nature of control systems, provides deeper insight.

\section{Phase transition}

Again, the essence of cognition is active choice. As described above, a cognitive system, confronted with uncertainty, must choose a small set of actual responses from a larger set of those available to it. Such choice reduces uncertainty, and the reduction of uncertainty implies the existence of an information source. The argument can be made quite formally (Wallace 2012, 2015a).

Given an information source associated with an inherently unstable, rapidfire cognitive $C^{3}$ system - said to be 'dual' to it - an equivalence class algebra emerges by choosing different system origin states $a_{0}$ and defining the equivalence of two subsequent states at times $m, n>0$, written as $a_{m}, a_{n}$, by the existence of high-probability meaningful paths connecting them to the same origin point. Disjoint partition by equivalence class, essentially similar to orbit equivalence classes in dynamical systems, defines a symmetry groupoid associated with the cognitive process. Groupoids represent generalizations of the group concept in which there is not necessarily a product defined for each possible element pair (Weinstein, 1996). The simplest example would be a disjoint union of groups.

The equivalence classes define a set of cognitive dual information sources available to the inherently unstable $C^{3}$ system, creating a large groupoid, with each orbit corresponding to a transitive groupoid whose disjoint union is the full groupoid. Each subgroupoid is associated with its own dual information source, and larger groupoids will have richer dual information sources than smaller.

Let $X_{G_{i}}$ be the $C^{3}$ dual information source associated with the groupoid element $G_{i}$, and let $Y$ be the information source associated with incoming environmental information, in a large sense See Wallace $(2012,2015 \mathrm{a})$ for details of how environmental regularities imply the existence of an environmental information source.

We construct a Morse Function (Pettini, 2007) as follows. 
Let $H\left(X_{G_{i}}, Y\right) \equiv H_{G_{i}}$ be the joint uncertainty of the two information sources. Define a Boltzmann-like pseudoprobability as

$$
P\left[H_{G_{i}}\right]=\frac{\exp \left[-H_{G_{i}} / \kappa \mathcal{H}\right]}{\sum_{j} \exp \left[-H_{G_{j}} / \kappa \mathcal{H}\right]}
$$

where $\kappa$ is an appropriate constant depending on the particular system and its linkages to control signals, and the sum is over the different possible cognitive modes of the full system.

A 'free energy' Morse Function $F$ can be defined as

$$
\exp [-F / \kappa \mathcal{H}] \equiv \sum_{j} \exp \left[-H_{G_{j}} / \kappa \mathcal{H}\right]
$$

Given the inherent groupoid structure, it is possible to apply an extension of Landau's picture of phase transition (Pettini, 2007). In Landau's formulation of spontaneous symmetry breaking, phase transitions driven by temperature changes occur as alteration of system symmetry, with higher energies at higher temperatures being more symmetric. The shift between symmetries is highly punctuated in the temperature index, here the minimum necessary control information rate $\mathcal{H}$ under the Data Rate Theorem for unstable control systems. Typically, such arguments involve only a very limited number of possible phases.

In this context, Birkhoff's (1960 p.146) perspective on the central role of groups in fluid mechanics is of considerable interest:

[Group symmetry] underlies the entire theories of dimensional analysis and modeling. In the form of 'inspectional analysis' it greatly generalizes these theories... [R]ecognition of groups... often makes possible reductions in the number of independent variables involved in partial differential equations... [E]ven after the number of independent variables is reduced to one... the resulting system of ordinary differential equations can often be integrated most easily by the use of group-theoretic considerations.

We will argue here that, for 'cognitive fluids' like vehicle traffic flows, groupoid generalizations of group theory become central.

Decline in the richness of control information $\mathcal{H}$, or in the ability of that information to influence the system, characterized by $\kappa$, can lead to punctuated decline in the complexity of cognitive process possible within the $C^{3}$ system, driving it into a ground state collapse that may not be actual instability' but rather a kind of dead zone in which, using the armed drone example, 'all possible targets are enemies'. This condition represents a dysfunctionally simple cognitive groupoid structure roughly akin to certain individual human psychopathologies (Wallace 2015a).

Below, we will argue that, for large-scale autonomous vehicle/intelligent infrastructure systems, the ground state dead zone involves massive, propagating tie-ups that far more resemble power network blackouts than traditional traffic 
jams. The essential feature is the role of vehicle road density $\rho$. Most of the topology of the inherently unstable vehicle/road system will be 'factored out' via the construction of geodesics in a topological quotient space, so that $\rho$ remains the only possible index of the rate of topological information generation for the DRT. Thus, in Eqs(4) and (5), $\mathcal{H}$ is replaced by the ratio $\mathcal{H} / f(\rho)$, where $f$ is a dimensionless monotonic increasing positive function.

For a fixed $\mathcal{H}$, increasing $\rho$ is then equivalent to lowering the 'temperature', and the system passes from high symmetry 'free flow' to different forms of 'crystalline' structure - broken symmetries representing platoons, shock fronts, traffic jams, and the like.

Again, making an exactly-solvable Black-Scholes approximation, the Mathematical Appendix shows that the 'cost' of the control information $\mathcal{H}$ can, in first order, be expressed in terms of $\rho$ as

$$
\mathcal{H} \approx \kappa_{1} \rho+\kappa_{2}
$$

where $\kappa_{i} \geq 0$. Again, in first order, taking $f(\rho) \approx \kappa_{3} \rho+\kappa_{4}>0$, we obtain an effective 'temperature' as

$$
\kappa \mathcal{H} / f(\rho) \approx \frac{\kappa_{1} \rho+\kappa_{2}}{\kappa_{3} \rho+\kappa_{4}}
$$

with limits $\kappa_{2} / \kappa_{4}, \rho \rightarrow 0$ and $\kappa_{1} / \kappa_{3}, \rho \rightarrow \infty$. Again, assuming $\kappa_{2} / \kappa_{4} \gg \kappa_{1} / \kappa_{3}$, increase in traffic density will quickly bring the effective 'temperature' below critical values, triggering collapse into a dysfunctional ground state, even though, in this case, the system might remain formally 'stable' under the Data Rate Theorem at the limit $\kappa_{1} / \kappa_{3}>0$. Thus we begin to explore variations in stability beyond the DRT itself.

The underlying dynamic can be treated in finer detail by viewing the initial phase transition as the first-order onset of a kind of 'turbulence', a transition from free flow to 'flock' structures like those studied in 'active matter' physics. Indeed, the traffic engineering perspective is quite precisely the inverse of mainstream active matter studies, which Ramaswamy (2010) describes as follows:

It is natural for a condensed matter physicist to regard a coherently moving flock of birds, beasts, or bacteria as an orientationally ordered phase of living matter. ...[M] odels showed a nonequilibrium phase transition from a disordered state to a flock with long-range order... in the particle velocities as the noise strength was decreased or the concentration of particles was raised.

In traffic engineering, the appearance of such 'long range order' is the first stage of a traffic jam (e.g., Kerner and Klenov 2009), a relation made explicit by Helbing (2001 Section VI) in his comprehensive review of traffic and related self-driven many-particle systems.

While flocking and schooling have obvious survival value against predation for animals in three-dimensional venues, long-range order - aggregation - among blood cells flowing along arteries is a blood clot and can be rapidly fatal. 


\section{Turbulence}

The 'free energy' function $F$ in equation (5) can be used to explore dynamics within a particular system phase defined by the associated groupoid.

Given a vector of system parameters $\mathbf{K}$, in standard manner it is possible to define an 'entropy' from $F$ as the Legendre transform

$$
S \equiv F(\mathbf{K})-\mathbf{K} \cdot \nabla_{\mathbf{K}} F
$$

and a nonequilibrium Onsager stochastic differential equation for dynamics in terms of the gradients in $S$ (de Groot and Mazur 1984), which can be written in one dimension as

$$
d K_{t}=-\left[\mu \partial S / \partial K_{t}\right] d t+\sigma K_{t} d B_{t}
$$

where $\mu$ is a diffusion coefficient. The last term represents a macroscopic volatility - proportional to the parameter $K$ - in which $d B_{t}$ is a noise term that may not be white, i.e., the quadratic variation $\left[B_{t}, B_{t}\right]$ may not be proportional to $t$. While details will depend on the particular circumstances, such systems are subject to a distressingly rich spectrum of possible instabilities (e.g., Khasminskii 2012). The full set of equations would involve properly indexed sums across the parameters making up the vector $\mathbf{K}$.

Allowing discontinuous Levy-like stochastic jumps, equation (9) can be solved explicitly, provided $K$ can be factored out and the equation set reexpressed as

$$
d K_{t}=K_{t-} d Y_{t}
$$

where $Y_{t}$ is a stochastic process, and $t$ - indicates left-continuous. Taking $\Delta Y_{t}=$ $Y_{t}-Y_{t-}$ as representing the jump process, the solution is in terms of the DoleansDade exponential (Protter 1990)

$$
K_{t}=\exp \left(Y_{t}-\frac{1}{2}\left[Y_{t}, Y_{t}\right]_{t}^{C}\right) \Pi_{s \leq t}\left(1+\Delta Y_{s}\right) \exp \left(-\Delta Y_{s}\right)
$$

where $\left[Y_{t}, Y_{t}\right]_{t}^{C}$ is the path-by-path continuous part of the quadratic variation of $Y_{t}$. This can be expressed

$$
\left[Y_{t}, Y_{t}\right]_{t}^{C}=\left[Y_{t}, Y_{t}\right]-\sum_{0 \leq s \leq t}\left(\Delta Y_{s}\right)^{2}
$$

The product term in equation (11), with jump processes having nonzero $\Delta$, converges.

Invoking the mean value theorem in equation (11), if, heuristically, $d Y_{t}<$ $1 / 2 d[Y, Y]_{t}^{C}$, then the expectation of $K, E(K)$, converges to zero. Otherwise, small perturbations will grow exponentially in expectation. This is an essential component of modern theories of turbulence (e.g., Ruelle 1983) and will prove central in understanding traffic flow instabilities. The phenomenon is roughly analogous to models of 'first order' phase transition, for example the sudden 
crystallization of a supercooled liquid when its container is tapped or a seed crystal is introduced. Higher order phase transitions involve discontinuities in higher order derivatives of system parameters and phase transitions may involve discontinuities at multiple 'derivative' scales.

A simple example. If a system following equation (10) has been initially placed in a characteristic eigenmode - e.g., the smooth part of a 'fundamental diagram' flow on some traffic network - then the dynamic equation for deviations in some parameter $K(t)$ from that mode can be written, in first order, as

$$
d K_{t} \approx a K_{t} d t+\sigma K_{t} d W_{t}
$$

where $d W_{t}$ represents white noise having uniform spectrum. Then, using the Ito chain rule,

$$
d \log [K]_{t} \approx\left(a-\sigma^{2} / 2\right) d t+\sigma d W_{t}
$$

The expectation is then

$$
E[K]_{t} \propto \exp \left[\left(a-\sigma^{2} / 2\right) t\right]
$$

so that, if $a<\sigma^{2} / 2, E[K] \rightarrow 0 . \sigma^{2}$ then - quite counterintuitively as described in Wallace (2016) - is a kind of control information in the sense of the Data Rate Theorem that serves to stabilize system dynamics. For an inherently unstable traffic flow system at low traffic density $\sigma^{2}=\omega \mathcal{H}$, where $\mathcal{H}$ is the minimum necessary control information to keep the vehicle on the road, and $\omega$ is the 'cost' of translation. Thus $\mathcal{H}$ represents the degree of independent control a driver or autonomous vehicle computer can exercise within local road flow, e.g., changing lanes, accelerating, slowing down, changing headway, predicting other vehicle maneuvers, detecting emerging bottlenecks, swerving, taking a different route, and so on.

Anticipating the argument, for traffic models below, we will argue that $\sigma^{2}$ must again be replaced by $\mathcal{H}(\rho) / g(\rho)$ where $g(\rho)$ is positive monontonic increasing. Then, antiparalleling the arguments of Belletti et al. (Bellitti et al. 2015, Section 2.3), for this simple example a 'traffic Froude number' (TFN) $\mathcal{F}$ that defines regimes of free and turbulent flow can be defined as

$$
\mathcal{F} \equiv 1-\left[a-\frac{1}{2}(\mathcal{H}(\rho) / g(\rho))\right]
$$

where $\mathcal{H}(\rho) / g(\rho)$ is clearly a variant of Eq.(7) and $a$ is $2 \alpha_{0}$ in Eq.(3).

When $\mathcal{F}>1$, the system is in 'laminar' free-flow, and becomes 'turbulent' when $\mathcal{F}<1$.

A more precise characterization, from this perspective, is that $\mathcal{H}(\rho) / g(\rho)$ represents a kind of viscosity index so that $\mathcal{F}$ is more akin to a Reynolds number than to a classical Froude number.

A difference between our approach and that of Bellitti et al. lies in the central object-of-interest. They invoke a hydrodynamic perspective involving the 'flow' of individual vehicles in a channel that finds 'instability' to be associated with unconstrained travel speed. The focus here is on the stability of geodesics in 
a complex topological quotient space $\mathcal{M}^{2 n} / W(r)$ that will be more precisely defined below. This is, in a sense, the inverse of their problem.

As argued at the end of Section 3, raising $\rho$ is equivalent to 'freezing' the system from 'liquid flow' to 'crystallized' broken symmetries - platoons, shock fronts, jams, and myriad other 'snowflake' fine structures.

$\left[Y_{t}^{j}, Y_{t}^{j}\right]_{t}^{C}$ may be further parameterized and, using the methods of Dzhaparidze and Spreij (1994), for colored noise, can be estimated from a time series data periodogram, as described in Wallace (2016). Increasing complexity in spectral structure is another marker of turbulence onset (Ruelle, 1983).

More specifically, for a stochastic process $X_{t}$ and a finite stopping time $T$, for each real number $\lambda$, the periodogram of $X$ at $T$ is defined as

$$
I_{T}(X ; \lambda) \equiv\left|\int_{0}^{T} \exp [i \lambda t] d X_{t}\right|^{2}
$$

Take $\epsilon$ as a real random variate that has a density function $r$ assumed to be symmetric around zero and examine, for any positive real number $L$,

$$
E_{\epsilon}\left[I_{T}(X ; L \epsilon]=\int_{-\infty}^{+\infty} I_{T}(X ; L s) r(s) d s\right.
$$

Some work shows that, for $L \rightarrow \infty$,

$$
E_{\epsilon}\left[I_{T}(X ; L \epsilon)\right] \rightarrow\left[X_{T}, X_{T}\right]
$$

Thus the quadratic variation can be estimated from observed time series data, as routinely done in financial engineering. Presumably, both high and low frequency limits could be explored in this method, parallel to what was done in Belletti et al., (2015).

\section{Information bottleneck}

Another modeling approach is via the information bottleneck method of Tishby et al. (1999). The essential idea is that the control information needed to stabilize an inherently unstable system, which we write as $\mathcal{H}$, can be used to define an average distortion measure in a rate distortion calculation. This involves an iterated application of the Rate Distortion Theorem (Cover and Thomas 2006) to a control system in which a series of 'orders' $y^{n}=y_{1}, \ldots, y_{n}$, having probability $p\left(y^{n}\right)$, is sent through and the outcomes monitored as $\hat{y}^{n}=\hat{y}_{1}, \ldots, \hat{y}_{n}$. The distortion measure is now the minimum necessary control information for system stability, $\mathcal{H}\left(y^{n}, \hat{y}^{n}\right)$. We can thus define an average 'distortion' $\hat{\mathcal{H}}$ as

$$
\hat{\mathcal{H}} \equiv \sum_{y^{n}} p\left(y^{n}\right) \mathcal{H}\left(y^{n}, \hat{y}^{n}\right) \geq 0
$$

It then is possible to define a new, iterated, Rate Distortion Function $\mathcal{R}(\hat{\mathcal{H}})$. 
For simplicity, we take $\mathcal{R}$ to be a Gaussian RDF in $\hat{\mathcal{H}}$,

$$
\begin{array}{r}
R(\hat{\mathcal{H}})=1 / 2 \log \left[\sigma^{2} / \hat{\mathcal{H}}\right] \hat{\mathcal{H}}<\sigma^{2} \\
R(\hat{\mathcal{H}})=0 \hat{\mathcal{H}} \geq \sigma^{2}
\end{array}
$$

Again, following Feynman (2000), information must be recognized as a form of free energy and a Rate Distortion Function can be used to define an 'entropy' as the Legendre transform

$$
\mathcal{S}=\mathcal{R}(\hat{\mathcal{H}})-\hat{\mathcal{H}} d \mathcal{R} / d \hat{\mathcal{H}}
$$

Taking Onsager's nonequilibrium thermodynamics perspective, the dynamics can, in first order, be characterized in terms of the gradients of $\mathcal{S}$, and we invoke an extended analog using the stochastic differential equation

$$
\begin{array}{r}
d \hat{\mathcal{H}}_{t}=\left[-\mu d \mathcal{S} / d \hat{\mathcal{H}}_{t}-F(\rho)\right] d t+\beta \hat{\mathcal{H}}_{t} d W_{t} \\
=\left[\frac{\mu}{2 \hat{\mathcal{H}}_{t}}-F(\rho)\right] d t+\beta \hat{\mathcal{H}}_{t} d W_{t}
\end{array}
$$

where $d W_{t}$ is standard white noise, and $F(\rho)$ is a function of traffic density $\rho$, the only possible determinant of the rate of generation of system 'topological information', given the extreme topological factoring associated with travel along a network. $\beta$ represents the magnitude of a 'volatility' noise term independent of $\sigma^{2}$ in the definition of $\mathcal{R}$ : higher $\mathcal{H}$, higher stochastic jitter.

Applying the Ito chain rule to the expectation of $\hat{\mathcal{H}}_{t}^{2}$, it becomes possible to explore the second moment stability of the system (Khashminskii 2012). A simple calculation finds that the expectation for $\hat{\mathcal{H}}^{2}$ cannot be a real number unless the discriminant of a quadratic equation is nonnegative, giving the necessary condition

$$
F(\rho) \geq \beta \sqrt{\mu}
$$

We force 'closure' to the model by taking $F(\rho)$ as given by Eq.(3), so that, again,

$$
\frac{\kappa_{1} \rho+\kappa_{2}}{\kappa_{3} \rho+\kappa_{4}} \geq \beta \sqrt{\mu} \equiv \alpha_{0}
$$

with similar restrictions on the constants $\kappa_{i}$ for stability.

Other channel forms will have analogous limits on traffic density as a consequence of the convexity of the RDF. The interested reader might carry the calculation through for the 'real' channel, having $\mathcal{R}(\hat{\mathcal{H}})=\sigma^{2} / \hat{\mathcal{H}}$.

\section{Reconsidering V2V/V2I systems}

Kerner et al. (2015) explicitly apply insights from statistical physics to traffic flow, writing 
In many equilibrium... and dissipative metastable systems of natural science... there can be a spontaneous phase transition from one metastable phase to another metastable phase of a system. Such spontaneous phase transition occurs when a nucleus for the transition appears randomly in an initial metastable phase of the system: The growth of the nucleus leads to the phase transition. The nucleus can be a fluctuation within the initial system phase whose amplitude is equal or larger than an amplitude of a critical nucleus required for spontaneous phase transition. Nuclei for such spontaneous phase transitions can be observed in empirical and experimental studies of many equilibrium and dissipative metastable systems... There can also be another source for the occurrence of a nucleus, rather than fluctuations: A nucleus can be induced by an external disturbance applied to the initial phase. In this case, the phase transition is called an induced phase transition...

A Data Rate Theorem approach to stability and flow of autonomous vehicle/traffic control systems, via spontaneous symmetry breaking in cognitive groupoids, generalizes and extends these insights, implying a far more complex picture of control requirements for inherently unstable systems than is suggested by the Theorem itself, or by 'physics' models of phase transition. That is, 'higher order' instabilities can appear. Such systems can require inordinate levels of control information. Here, we find that $C^{3}$ systems may remain 'stable' in the strict sense of the DRT, but can collapse into a ground state analogous to certain psychopathologies, or, following the arguments above, into even more complicated pathological dynamics. In biological circumstances, such failures can be associated with the onset of senescence (Wallace 2014, 2015b). Apparently, rapidly responding, and hence almost certainly inherently unstable, $C^{3}$ systems can display recognizable analogs to senility under fog-of-war demands.

Using these ideas, it becomes possible to formally represent the interaction of cognitive ground state collapse in autonomous vehicle/intelligent road systems with critical transitions in traffic flow.

Recall that, defining 'stability' as the ability to return, after perturbation, to the streamline geodesic trajectory of the embedding, topologically complex, road network, it is clear that individual autonomous vehicles are inherently unstable and require a constant flow of control information for safe operation, unlike aircraft that can, in fact, be made inherently stable by placing the center of pressure well behind the center of gravity. There is no such configuration possible for ground-based vehicles following sinuous road geometries, particularly in heavy traffic.

The argument can be made more precise using the approach of $\mathrm{Hu}$ et al. (2001) who show that, in the context of air traffic control, finding collision-free maneuvers for multiple agents on a Euclidean plane surface $\mathcal{R}^{2}$ is the same as finding the shortest geodesic in a particular manifold with nonsmooth boundary. Given $n$ vehicles, the geodesic is calculated for the quotient space $\mathcal{R}^{2 n} / W(r)$, where $W(r)$ is defined by the requirement that no vehicles are closer together 
than some critical Euclidean distance $r$. For autonomous ground vehicles, $\mathcal{R}^{2}$ must be replaced by a far more topologically complex roadmap space $\mathcal{M}^{2}$ subject to traffic jams and other 'snowflake' condensation geometries in real time. Geodesics for $n$ vehicles are then in a quotient space $\mathcal{M}^{2 n} / W(r)$ whose dynamics are subject to phase transitions in vehicle density $\rho$ (Kerner and Klenov, 2009; Jin et al., 2013) that represent cognitive groupoid symmetry breaking. Recall figure 1. Again, the vertical axis shows the number of vehicles per hour, the horizontal, the density of vehicles per mile. The streamline geodesic flow, and deviations from it at critical vehicle density, are evident. Some of the phases may be 'supercooled' - fast-flowing 'liquid' at higher-than-critical densities. Additional 'fine structure' should be expected within both geodesic and turbulent modes.

Again, given the factoring out of most of the topological structure by the construction of geodesics in the quotient space $\mathcal{M}^{2 n} / W(r)$, the only parameter available to represent the rate of generation of topological information in the inherently unstable traffic flow system is the vehicle density $\rho$.

Classic traffic flow models based on extensions of hydrodynamic perspectives involving hyperbolic partial differential equations (HPDE's) can be analogously factored using the methods of characteristic curves and Riemann invariants - streamlines (e.g., Landau and Lifshitz 1987). Along characteristic curves, HPDE's are projected down to ordinary differential equations (ODE's) that are usually far easier to solve. The ODE solution or solutions can then be projected upward as solutions to the HPDE's. Here, reduction involves expressing complex dynamics in terms of relatively simple stochastic differential equations and their stability properties. Those stability properties, marking the onset of 'turbulence', will be of central interest.

Taking a somewhat larger view, cognitive phase transitions in autonomous vehicle systems, in particular ground state collapse to some equivalent of 'all possible targets are enemies', should become synergistic with traffic flow phase transitions to produce truly monumental traffic jams, and it is possible to model this phenomenon, to first order, in terms of spontaneous symmetry breaking on groupoids.

Consider a random network of roads between nodal points - intersections. If the average probability of passage falls below a critical value, the Erdos/Renyi 'giant component' that connects across the full network breaks into a set of disjoint connected equivalence class subcomponents, with 'bottlenecks' at which traffic jams occur marking corridors between them. Li et al. (2015), in fact, explicitly apply a similar percolation model to explain this effect for road congestion in a district of Beijing. The underlying road network is shown in figure 2 , and in figure 3 a cross section taken during rush hour showing disjoint sections when regions with average velocity below $40 \%$ of observed maximum for the road link have been removed.

Such equivalence classes define a groupoid (Weinstein, 1996). Above, we defined the cognitive groupoid to be associated with a $C^{3}$ structure, here a system of autonomous vehicles linked together in a V2V 'swarm intelligence' embedded in a larger vehicle to infrastructure (V2I) traffic management system. Individ- 


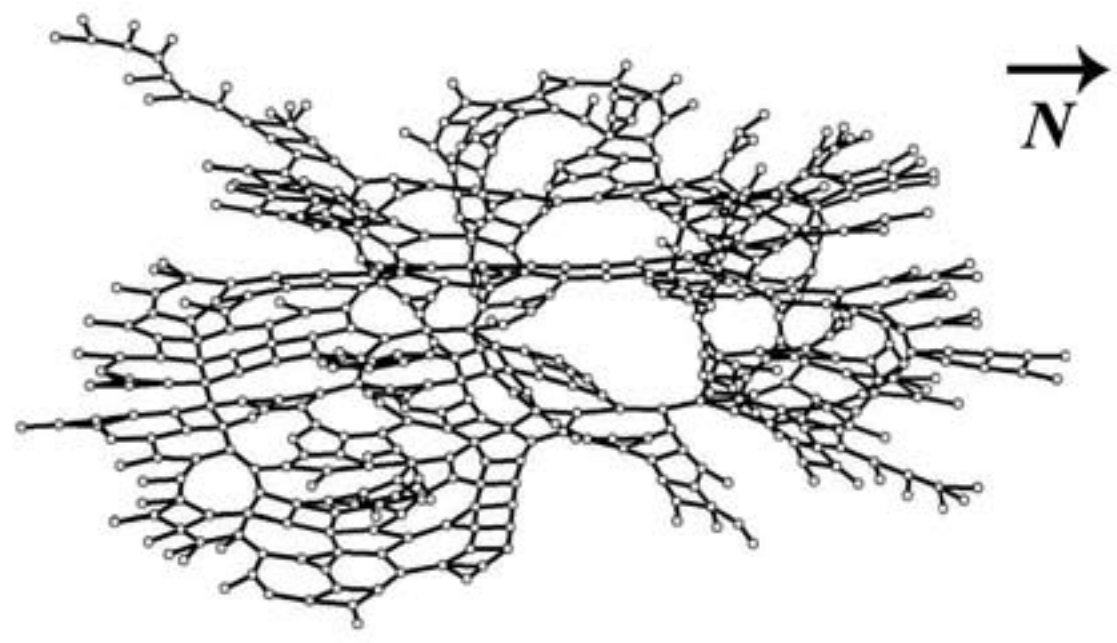

Figure 2: Adapted from Li et al. (2015). Full road network near central Beijing.

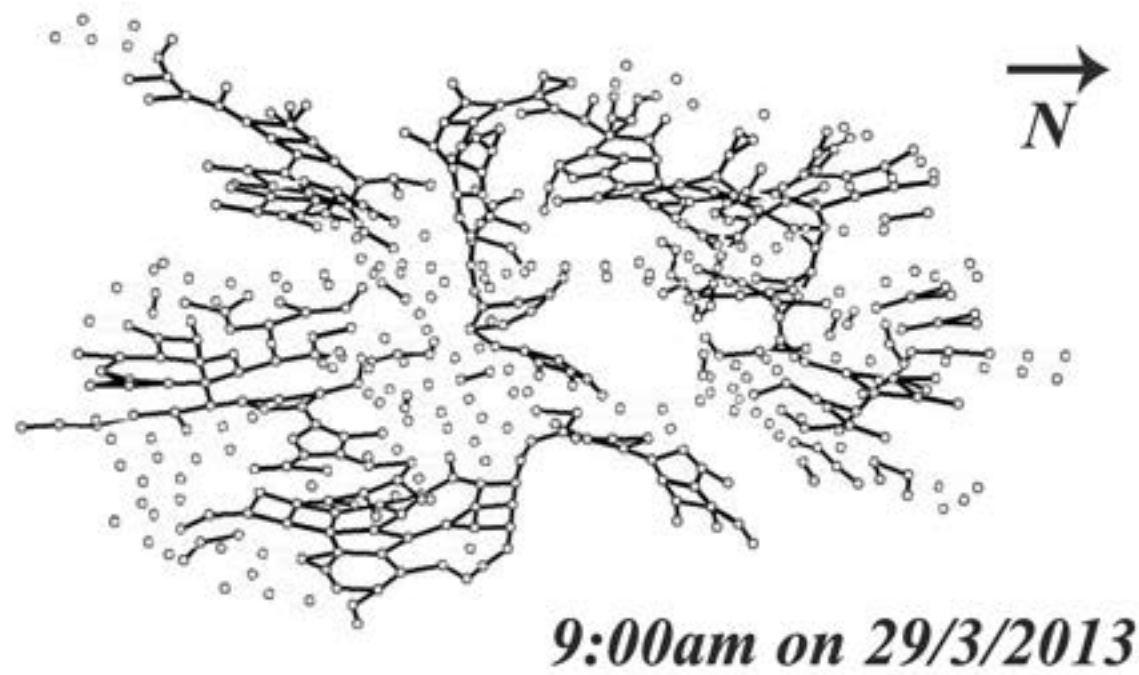

Figure 3: Adapted from Li et al., (2015). Disconnected subcomponents of Beijing central road network at rush hour. Sections with average vehicle velocity less than $40 \%$ of maximum observed have been removed. 
ual vehicle spacings, speed, acceleration, lane-change, and so on are determined by this encompassing distributed cognitive machine that attempts to optimize traffic flow and safety. The associated individual groupoids are the basic transitive groupoids that build a larger composite groupoid. Thus, under declining probability of passage, related to traffic congestion and viewed here as a temperature analog, this 'vehicle/road' groupoid undergoes a symmetry-breaking transition into a combined cognitive ground state collapse and traffic jam mode - essentially a transition from 'laminar' geodesic to 'turbulent' or 'crystallized' flow. Autonomous vehicle systems that become senile under fog-of-war demands will likely trigger traffic jams that are far different from those associated with human-controlled vehicles. There is no reason to believe that such differences will be benign.

A more precise-seeming model can be built using the turbulence limit of equations (9) and (10), under conditions of white noise, so that $[Y, Y]^{C} \propto t$, where $t$ is the time.

Following figure 1, suppose the system has been placed in a geodesic for the map quotient space $\mathcal{M}^{2 n} / W(r)$, and is free-flowing 'laminar' at some vehicle density $\rho$, but may be inherently unstable. As described above, for the simplest kinds of stochastic differential equation models involving white noise - equation (14) - heuristically,

$$
\begin{array}{r}
Y_{t} \rightarrow a t \\
{\left[Y_{t}, Y_{t}\right] \rightarrow(\mathcal{H}(\rho) / g(\rho)) t}
\end{array}
$$

in the exponential of equation (11), where $\alpha>0$ and $\mathcal{H}$ represents the degree of 'driver' control information necessary to keep a vehicle on the road according to the DRT. As argued, this may include lane change, speed variation, spacing change, and other maneuvers.

Again, in first order, if

$$
a-1 / 2(\mathcal{H}(\rho) / g(\rho))<0
$$

then any perturbation $K$ dies out in expectation - the driver/autonomous vehicle can exercise sufficient initiative to damp out occasional glitches - and the system has sufficient symmetry so that it can return to streamline geodesic flow. If $\rho$ increases beyond a critical value, then too limited spacing constrains the possibility of cognitive vehicle initiative, the 'temperature' falls below criticality, and perturbations grow exponentially in time so that instability causes 'turbulent' crystal formation - 'fine structure' traffic jams of one form or another. As argued above, this can happen even though the system remains fully 'stable' under the Data Rate Theorem: the individual vehicle control information rate $\mathcal{H}(\rho)$ remains sufficient so that no crash occurs. A central concept of modern theories of turbulence is the onset of exponential growth in small perturbations (Ruelle, 1983).

The analysis of traffic flow on a network is, conceptually, somewhat similar to characterizing the propagation of a 'traffic jam signal' via the Markov 'network 
dynamics' formalism of (Wallace, 2016; Gould and Wallace, 1994), a method that might be used to empirically identify geodesic eigenmodes of real road network systems under different conditions, as opposed to individual vehicle dynamics or flow on a single road. Abducting the approach of Gould and Wallace (1994), the spread of a 'signal' on a particular network of interacting sites between and within - is described at nonequilibrium steady state in terms of an equilibrium distribution $\epsilon_{i}$ 'per unit area' $A_{i}$ of a Markov process, where $A$ scales with the different 'size' of each node, taken as distinguishable by a scale variable $A$ (for example number of entering streets or average total traffic flow) as well as by its 'position' $i$ or the associated probability-of-contact matrix (POCM). The POCM is normalized to a stochastic matrix $\mathbf{Q}$ having unit row sums, and the vector $\epsilon$ calculated as $\epsilon=\epsilon \mathbf{Q}$

There is a vector set of dimensionless network flows $\mathcal{X}_{t}^{i}, i=1, \ldots, n$ at time $t$. These are each determined by some relation

$$
\mathcal{X}_{t}^{i}=f\left(t, \epsilon_{i} / A_{i}\right)
$$

Here, $i$ is the index of the node of interest, $\mathcal{X}_{t}^{i}$ is the corresponding dimensionless scaled i-th signal, $t$ the time, and $f$ an appropriate function. Again, $\epsilon_{i}$ is defined by the relation $\epsilon=\epsilon \mathbf{Q}$ for a stochastic matrix $\mathbf{Q}$, calculated as the network probability-of-contact matrix between regions, normalized to unit row sums. Using $\mathbf{Q}$, we have broken out the underlying network topology, a fixed between-and-within travel configuration weighted by usage that is assumed to change relatively slowly on the timescale of observation compared to the time needed to approach the nonequilibrium steady state distribution.

Since the $\mathcal{X}$ are expressed in dimensionless form, $f, t$, and $A$ must be rewritten as dimensionless as well giving, for the monotonic increasing (or thresholdtriggered) function $F$

$$
\mathcal{X}_{\tau}^{i}=F\left[\tau, \frac{\epsilon_{i}}{A_{i}} \times \mathcal{A}_{\tau}\right]
$$

where $\mathcal{A}_{\tau}$ is the value of a 'characteristic area' variate that represents the spread of the perturbation signal - evolving into a traffic jam under worst-case conditions - at (dimensionless) characteristic time $\tau=t / T_{0}$.

$F$ may be quite complicated, including dimensionless 'structural' variates for each individual geographic node $i$. The idea is that the characteristic 'area' $\mathcal{A}_{\tau}$ grows according to a stochastic process, even though $F$ may be a deterministic mixmaster driven by systematic local probability-of-contact or flow patterns. Then the appropriate model for $\mathcal{A}_{\tau}$ of a spreading traffic jam becomes something like equations (9) or (10), with $K$ replaced by $\mathcal{A}$ and $t$ by $\tau$. Thus, for the network, the signal $Y_{\tau}$ must again have a 'noise'/vehicle density threshold condition like Eq.(16) for large-scale propagation of a traffic jam across the full network - something that would look very similar to the spread of a power blackout.

Zhang (2015) uses a similar Markov method to examine taxicab GPS data for transit within and between 12 empirically-identified 'hot zones' in Shanghai, determining the POCM and its equilibrium distribution. 
This approach is something in the spirit of a long line of work summarized by Cassidy et al. (2011) that attempts to extend the idea of a fundamental diagram for a single road to a full transport network. As they put it,

Macroscopic fundamental diagrams (MFDs)... relate the total time spent to the total distance traveled... It is proposed that these macrolevel relations should be observed if the data come from periods when all lanes on all links throughout the network are in either the congested or the uncontested regime...

Following our arguments here, such conditions might apply when $\mathcal{A}_{\tau} \rightarrow 0$, or when it encompasses the entire network domain. Indeed, figure 3 suggests why MFDs cannot be constructed in general: congested and free flowing sections of traffic networks will often, and perhaps usually, coexist in an essentially random manner depending on local traffic densities. Figure 4, adapted from Geroliminis and Sun (2011), shows the limitations of the MFD approach. It examines the flow, in vehicles $/ 5 \mathrm{~min}$ intervals, vs. percent occupancy over a three day period for the Minnesota Twin Cities freeway network that connects St. Paul and Minneapolis. See figure 1 of their paper for details of the road and sensor spacing. Evidently, while the unconstrained region of occupancy permits characterization of a geodesic mode, both strong hysteresis and phase transition effects are evident after about $8 \%$ occupancy, analogous to the 'nucleation' dyanamics of figure 1 at high traffic density. Again, as in figure 1 'fine structure' should be expected within both geodesic and turbulent modes, depending on local parameters.

Daganzo et al. (2010) further find that MFD flow, when it can be characterized at all, will become unstable if the average network traffic density is sufficiently high. They find that, for certain network configurations, the stable congested state

...is one of complete gridlock with zero flow. It is therefore important to ensure that in real-world applications that a network's [traffic] density never be allowed to approach this critical value.

Daqing et al. (2014) examine the dynamic spread of traffic congestion on the Beijing central road network. They characterize the failure of a road segment to be a traffic velocity less than $20 \mathrm{~km} / \mathrm{hr}$ and use observational data to define a spatial correlation length in terms of the Euclidean distances between failed nodes. Our equivalent might be something like $\sqrt{\mathcal{A}_{\tau}}$. Adapting their results, figure 5 shows the daily pattern of the correlation length of cascading traffic jams over a 9 day period. The two commuting maxima are evident, and greatest correlation lengths reach the diameter of the main part of the city. Even at rush hour, no MFD can be defined, as, according to figure 3, the network will be a dynamic patchwork of free and congested components.

A next step would be to allow $\rho$ vary in space and time, i.e., to parameterize the model using the moments of vehicle density.

Figure 6, adapted from Rand (1979, figure 6.4), provides a disturbing counterexample to these careful empirical and theoretical results on network traffic 


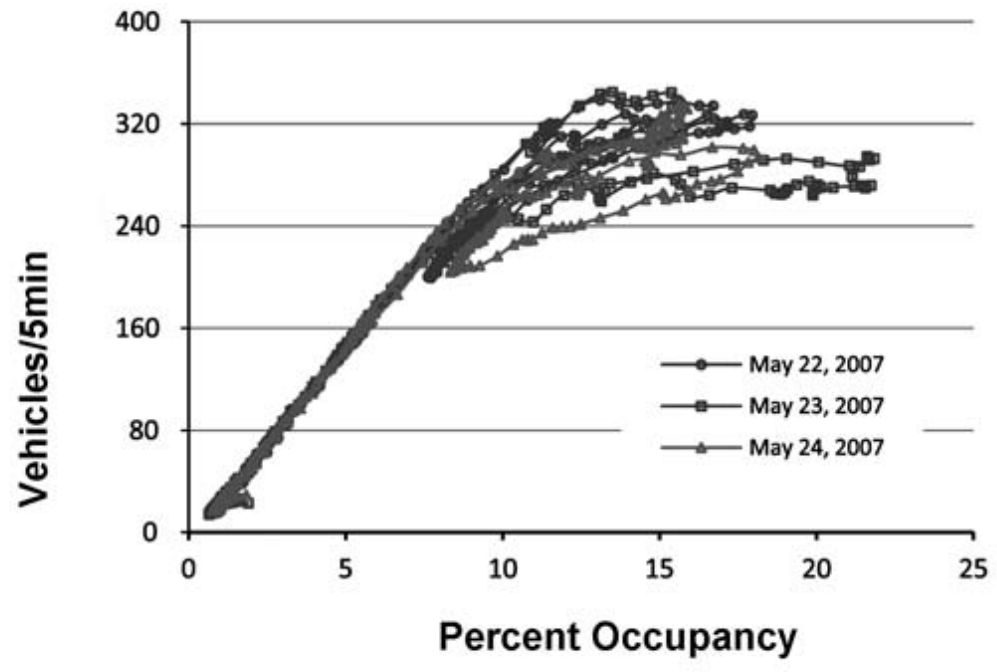

Figure 4: Adapted from Geroliminis and Sun (2011). Breakdown of the macroscopic fundamental diagram for the freeway network connecting St. Paul and Minneapolis at high vehicle densities. Both nucleation and hysteresis effects are evident, showing the fine structure within the turbulent mode. 


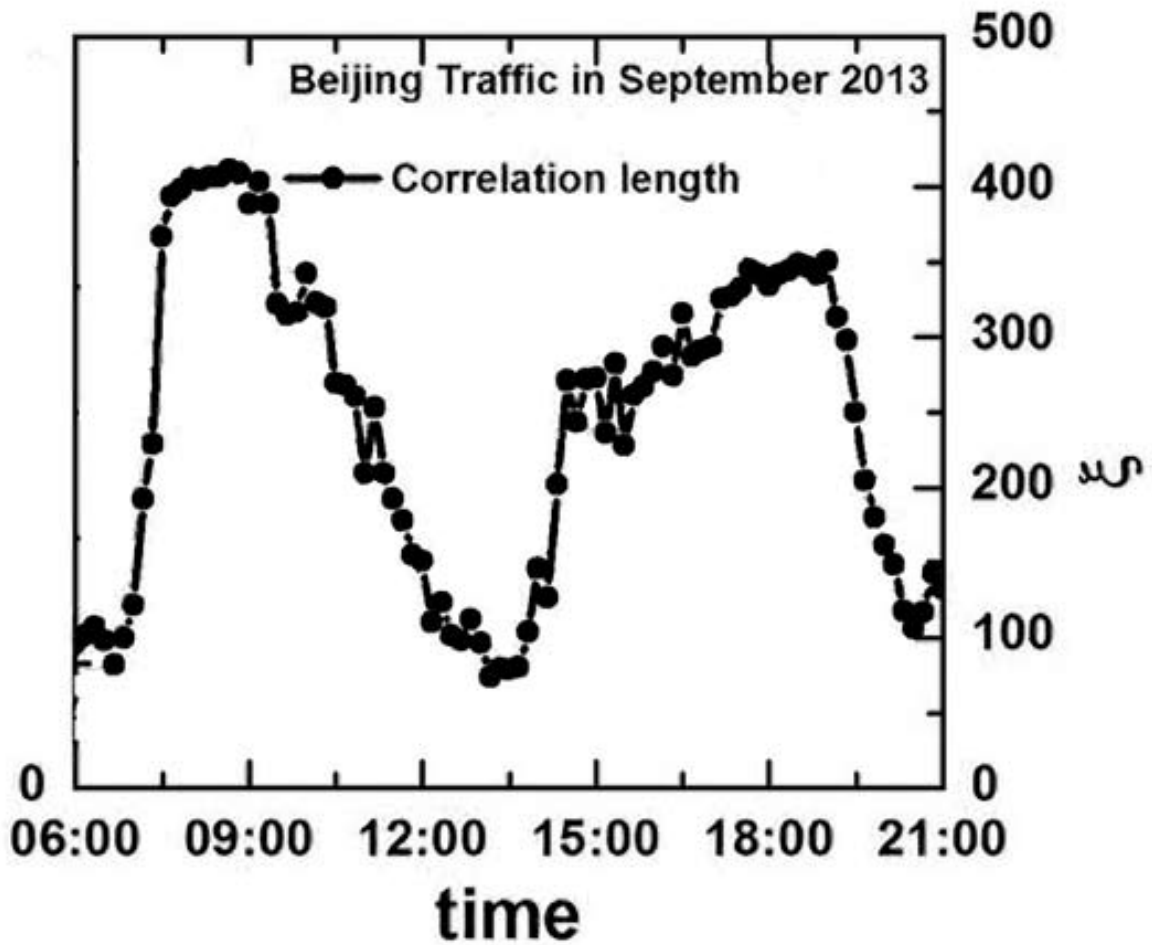

Figure 5: Adapted from Daqing et al. (2014). Daily cycle of traffic jam correlation length over a 9 day period in central Beijing. The maxima cover most of the central city. Even for rush hour, however, no macroscopic fundamental diagram can be defined since the region is characterized by a patchwork of free and congested parts, as shown in figure 3. 
flow, one with unfortunate results. Summarizing observations carried out by the Rand Fire Project, it represents a repeated sampling of 'travel time vs. distance' for the full Trenton NJ road network in 1975 under varying conditions of time-of-day, day-of-week, weather, and so on, by fire companies responding to calls for service. This was an attempt to create a Macroscopic Fundamental Diagram in the sense used above, but without any reference at all to traffic density.

Indeed, fire service responses are a traffic flow 'best case' as fire units are permitted to bypass one-way restrictions, traffic lights, and so on, and usually able to surmount even the worst weather conditions. In spite of best-case circumstances, the scatterplot evidently samples whole-network turbulent flow, not unlike that to the right of the local geodesic in figures 1 and 4 , part of a single street and a highway network, respectively, and consistent with the assertions of Cassidy et al. (2011) that MFD relations can only be defined under very restrictive conditions, i.e., either complete free flow or full network congestion.

The Rand Fire Project, when confronted with intractable whole-network traffic turbulence, simply collapsed the data onto a 'square root-linear' relation, as indicated on the figure. The computer models resulting from this gross oversimplification were used to determine fire service deployment strategies for high fire incidence, overcrowded neighborhoods in a number of US cities, with literally devastating results and consequent massive impacts on public health and public order. Wallace and Wallace (1998), produced under an Investigator Award in Health Policy Research from the Robert Wood Johnson Foundation, documents the New York City case history. The Rand models are still in use by the New York City Fire Department, for political purposes outlined in that analysis.

\section{Discussion and conclusions}

Ruelle (1983), in his elegant keynote address on turbulent dynamics, raises a red flag for any traffic flow studies:

...[A] deductive theory of developed turbulence does not exist, and a mathematical basis for the important theoretical literature on the subject is still lacking... A purely deductive analysis starting with the Navier-Stokes equation... does not appear feasible... and might be inappropriate because of the approximate nature of the... equation.

Or, as the mathematician Garrett Birkoff (1960 p.5) put it, “...[V]ery few of the deductions of rational hydrodynamics can be established rigorously".

Similar problems afflict the exactly solvable but highly approximate BlackScholes models of financial engineering, and institutions that rely heavily on them have gone bankrupt in the face of market turbulence (Wallace 2015c).

Turbulence in traffic flow does not represent simple drift from steady linear or even parallel travel trajectories. Traffic turbulence involves the exponential 


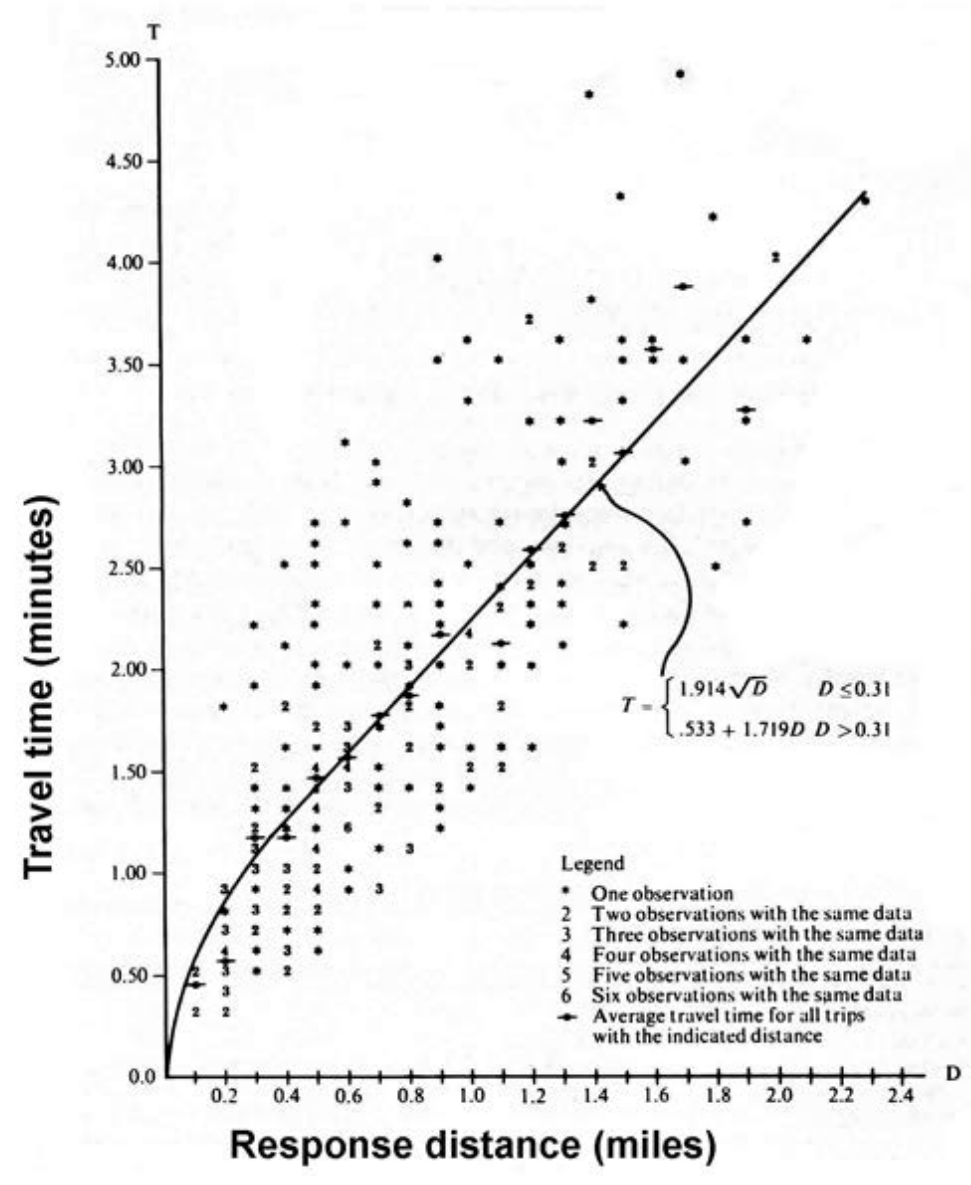

Figure 6: Adapted from figure 6.4 of Rand (1979). Relation between fire company travel time and response distance for the full Trenton, NJ road network, 1975. The Rand Fire Project collapsed evident large-scale traffic turbulence into a simple 'square root-linear' model used to design fire service deployment policies in high fire incidence, high population density neighborhoods of many US cities, including the infamous South Bronx. The impacts were literally devastating (Wallace and Wallace, 1998). 
amplification of small perturbations into large-scale deviations from complicated streamline geodesics in a topologically complex map quotient space. This is the mechanism of groupoid 'symmetry breaking' by which the system undergoes a phase transition from 'liquid' geodesic flow to 'crystalline' phases of shock fronts, platoons, and outright jams. Under such circumstances, cognitive vehicle initiative - the stabilizing control information we have called $\mathcal{H}$ - serves as a mechanism for returning to local geodesic flow. Inhibition of cognitive initiative occurs when vehicle density exceeds a critical limit, triggering complex dynamic condensation patterns and, for autonomous vehicle systems, perhaps even more disruptive behaviors.

It is, then, not enough to envision atomistic autonomous ground vehicles as having only local dynamics in an embedding traffic stream, as seems the current American and European practice. Traffic light strategies, a frequentlyshifting road map space, and the dynamic composition of the traffic stream, all create the synergistic context in which individual vehicles operate and which constitutes the individual 'driving experience'. It is necessary to understand the dynamics of that full system, not simply the behavior of a vehicle atom within it, and the properties of that system will be both overtly and subtly emergent, as will, we assert, the responses of cognitive vehicles enmeshed in context, whether controlled by humans or machines.

One inference from this analysis is that failure modes afflicting large-scale $\mathrm{V} 2 \mathrm{~V} / \mathrm{V} 2 \mathrm{I}$ systems are likely to be more akin to power blackouts than to traffic jams as we know them, and the description by Kinney et al. (2005) is of interest:

Today the North American power grid is one of the most complex and interconnected systems of our time, and about one half of all domestic generation is sold over ever-increasing distances on the wholesale market before it is delivered to customers... Unfortunately the same capabilities that allow power to be transferred over hundreds of miles also enable the propagation of local failures into grid-wide events... It is increasingly recognized that understanding the complex emergent behaviors of the power grid can only be understood from a systems perspective, taking advantage of the recent advances in complex network theory...

Dobson (2007) puts it as follows:

$[\mathrm{P}]$ robabalistic models of cascading failure and power system simulations suggest that there is a critical loading at which expected blackout size sharply increases and there is a power law in the distribution of blackout size... There are two attributes of the critical loading: 1. A sharp change in gradient of some quantity such as expected blackout size as one passes through the critical loading. 2. A power law region in probability distribution of blackout size at the critical loading. We use the terminology 'critical' because this behavior is analogous to a critical phase transition in statistical physics. 
Daqing et al. (2014), in fact, explicitly link traffic jams and power failures:

Cascading failures have become major threats to network robustness due to their potential catastrophic consequences, where local perturbations can induce global propagation of failures... [that] propagate through collective interactions among system components.... [W]e find by analyzing our collected data that jams in city traffic and faults in power grid are spatially long-range correlated with correlations decaying slowly with distance. Moreover, we find in the daily traffic, that the correlation length increases dramatically and reaches maximum, when morning or evening rush hour is approaching...

While clever V2V/V2I management strategies might keep rush hour traffic streams in supercooled high-flow mode beyond critical densities, such a state is notoriously unstable. More subtle patterns of autonomous vehicle 'psychopathology' may be even less benign.

\section{Mathematical Appendix}

Suppose the system is stable, in the control theory sense, and take $\mathcal{H}$ as the 'cost' of stability at the traffic density $\rho$. What is the form of $\mathcal{H}(\rho)$ under conditions of volatility i.e., variability in $\rho$ proportional to it? Let

$$
d \rho_{t}=g\left(t, \rho_{t}\right) d t+b \rho_{t} d W_{t}
$$

where $d W_{t}$ is taken as white noise.

Assuming stability, let $\mathcal{H}\left(\rho_{t}, t\right)$ be the minimum needed incoming rate of control information under the Data Rate Theorem, and expand in $\rho$ using the Ito chain rule

$$
\begin{aligned}
d \mathcal{H}_{t}=\left[\partial \mathcal{H} / \partial t+g\left(\rho_{t}, t\right) \partial \mathcal{H} / \partial \rho+\right. & \left.\frac{1}{2} b^{2} \rho_{t}^{2} \partial^{2} \mathcal{H} / \partial \rho^{2}\right] d t \\
& +\left[b \rho_{t} \partial \mathcal{H} / \partial \rho\right] d W_{t}
\end{aligned}
$$

We define a quantity $L$ as a Legendre transform of the rate $\mathcal{H}$, by convention having the form

$$
L=-\mathcal{H}+\rho \partial \mathcal{H} / \partial \rho
$$

Since $\mathcal{H}$ is an information index, it is a kind of free energy in the sense of Feynman (2000) and $L$ is a classic entropy measure.

Heuristically, replacing $d X$ with $\Delta X$ in these expressions and applying Eq.(30) gives

$$
\Delta L=\left(-\partial \mathcal{H} / \partial t-\frac{1}{2} b^{2} \rho^{2} \partial^{2} \mathcal{H} / \partial \rho^{2}\right) \Delta t
$$

As in the classical Black-Scholes model (Black and Scholes 1973), the terms in $g$ and $d W_{t}$ cancel out, and the effects of noise are subsumed into the Ito 
correction factor, a powerful regularity assumption that makes this an exactly solvable approximate model.

The conventional Black-Scholes calculation takes $\Delta L / \Delta T \propto L$. Here, at nonequilibrium steady state, we assume $\Delta L / \Delta t=\partial \mathcal{H} / \partial t=0$, so that

$$
-\frac{1}{2} b^{2} \rho^{2} \partial^{2} \mathcal{H} / \partial \rho^{2}=0
$$

By inspection,

$$
\mathcal{H}=\kappa_{1} \rho+\kappa_{2}
$$

where the $\kappa_{i}$ are nonnegative constants.

\section{Acknowledgments}

The author thanks Dr. D.N. Wallace for useful discussions.

\section{References}

F. Belletti, M. Huo, X. Litrico, A. Bayen, Prediction of traffic convective instability with spectral analysis of the Aw-Rascle-Zhang model, Phys. Lett. A, 379:2319-2330, 2015.

G. Birkoff, Hydrodynamics: A study in logic, fact, and similitude, Second Edition, Princeton University Press, Princeton, NJ, 1960.

F.Black, M. Scholes, The pricing of options and corporate liabilities, J Pol Econ 81:637-654 1973.

S. Blandin et al., A general phase transition model for vehicular traffic, SIAM J Appl Math 71:107-127, 2011.

G. Box, N. Draper, Empirical Model-Building and Response Surfaces, Wiley New York, 1987.

M. Cassidy, K. Jang, C. Daganzo, Macroscopic fundamental diagrams for freeway networks, Trans. Res. Rec. 2260:8-15, 2011.

L. Daqing, J. Yinan, K. Rui, S. Havlin, Spatial correlation analysis of cascading failures: congestions and blackouts, Sci. Reports 4:5381, 2014.

S. de Groot, P. Mazur, Nonequilibrium Thermodynamics, Dover, New York, 1984.

I. Dobson, Where is the edge for cascading failure?: challenges and opportunities for quantifying blackout risk, IEEE Power Eng. Soc. General Meeting, Tampa Fl. USA (dobson@engr.wisc.edu), 2007.

C. Dzganzo, V. Gayah, E. Gonzales, Macroscopic relations of urban traffic variables: an analysis of instability. Working Paper UCB-ITS-VWP-2010-4, UC Berkeley Center for Future Urban Transit, 2010.

K. Dzhaparidze, P. Spreij, Spectral characteristics of the optional quadratic variantion process, Stochastic Processes and their Applications, 54:165-174, 1994. 
N. Geroliminis, J. Sun, Properties of a well-defined macroscopic fundamental diagram for urban traffic, Trans. Res. B 45:605-617, 2011.

P. Gould, R. Wallace, Spatial structures and scientific paradoxex in the AIDS pandemic, Geograph. Ann. 76B:105-116, 1994.

D. Helbing, Traffic and related self-driven many-particle systems, Rev. Mod. Phys. 73:1067-1141, 2001.

J. Hu, M. Prandini, K. Johnasson, S. Sastry, Hybrid geodesics as optimal solutions to the collision-free motion planning problem. In M. Di Benedetto, A. Sangiovanni-Vincentelli (Eds.) HSCC 2001, LNCS 2034:305-318, 2001.

C. Jin et al., Spontaneous phase transition from free flow to synchronized flow in traffic on a single-lane highway, Phys. Rev. E. 87:012815, 2013.

B. Kerner, S. Klenov, Phase transitions in traffic flow on multilane roads, Phys. Rev. E 80:056101, 2009.

B. Kerner, M. Koller, S. Klenov, H. Rehborn, M. Leibel, Empirical features of spontaneous and induced traffic breakdowns in free flow at highway bottlenecks, ArXiv:1502.02862v2 [physics.soc-ph], 2015.

R. Khasminskii, Stochastic Stability of Differential Equations, 2nd Edition, Spriner, New York, 2012.

R. Kinney et al., Modeling cascading failures in the North American power grid, Eur. Phys. J. B. 46:101-107, 2005.

L. Landau, E. Lifshitz, Fluid Mechanics, Second Edition, Pergamon Press, NY, 1987.

D. Li, B. Fu, Y. Wang, G. Lu, Y. Berezin, H.E. Stanley, Percolation transition in dynamical traffic network with evolving critical bottlenecks, PNAS, 112:669-672, 2015.

G. Nair, F. Fagnani, S. Zampieri, R. Evans, Feedback control under data rate constraints: an overview, Proc. IEEE 95:108-137, 2007.

G. Orosz, R.E. Wilaon, G. Stepan, Traffic jams: dynamics and control, Phil. Trans. R. Soc. A 368:4455-4479, 2010.

M. Pettini, Geometry and Topology in Hamiltonian Systems, Springer, New York, 2007.

P. Protter, Stochastic Integration and Differential Equations, Springer, New York, 1990.

S. Ramaswamy, The mechanics and statistics of active matter, Ann. Rev. Condens. Matter Phys., 1:323-345, 2010.

The Rand Fire Project, Fire Deployment Analysis: A public policy analysis case study, North Holland, New York, 1979.

D. Ruelle, Turbulent dynamical systems, Proceedings of the International Congress of Mathematicians, Aug. 16-24, Warsaw, pp. 271-286, 1983.

N. Tishby, F. Pereira, W. Bialek, The information bottleneck method, 37th Allerton Conference on Communication, Control and Computing, pp.368-377, 1999.

D. Wallace, R. Wallace, A Plague on Your Houses, Verso, New York, 1998.

R. Wallace, Consciousness, crosstalk, and the mereological fallacy: an evolutionary perspective, Phys. Life. Rev. 9:426-453, 2012. 
R. Wallace, Extending Swerdlow's hypothesis: statistical models of mitochondrial deterioration and aging, J. Math. Chem. 52:2663-2679, 2014.

R. Wallace, Closed-system 'economic' models for psychiatric disorders: Western atomism and its culture-bound syndromes, Cogn. Process. 16:279-290, 2015a.

R. Wallace, An Information Approach to Mitochondrial Dysfunction, World Scientific, Singapore, 2015b.

R. Wallace, An Ecosystem Approach to Economic Stabilization: Escaping the neoliberal wilderness, Routledge Advances in Heterodox Economics, London, 2015c.

R. Wallace, Subtle noise structures as control signals in high-order biocognition, Phys. Let. A, doi:10.1016/j.physleta.2015.11.037, 2016.

A. Weinstein, Groupoids: unifying internal and external symmetry, Not. Am. Math. Soc. 43:744-752, 1996.

L. Zhang, Realistic, efficient and secure geographic routing in vehicular networks, PhD Thesis in the Department of Computer Science, University of Victoria (CA), 2015. 